\title{
Center/Surround Retinex: Analysis and Implementation
}

\author{
Jose-Luis Lisani, Ana-Belén Petro, Catalina Sbert \\ Universitat Illes Balears, Spain \\ \{joseluis.lisani, anabelen.petro, catalina.sbert\}@uib.es \\ Communicated by Jean-Michel Morel Demo edited by Jose-Luis Lisani
}

\begin{abstract}
The Retinex perception theory tries to mimic the human ability to cope with the high dynamic range of natural scenes. In 1986 E. Land proposed a formulation of this model in terms of a Center/Surround operation involving two steps, a local adaptation and a global transform. This model gave rise to the so-called Center/Surround tone-mapping algorithms. In this paper we unify the different Center/Surround algorithms proposed in the literature using a common framework and analyze several possibilities for the local and global operations involved.
\end{abstract}

\section{Source Code}

The reviewed and documented source code and an online demo are available at the web page of this article ${ }^{1}$. Compilation and usage instructions are included in the README.txt file of the archive.

Keywords: Retinex theory; tone mapping; center/surround method; color enhancement

\section{Introduction}

The adaptation of the human visual system (HVS) to several orders of magnitude of light intensity permits us to perceive a wide spectrum of lights and contrasts which, in general, a camera is unable to capture. The reason is that the eye is a contrast detector, not an absolute detector like the sensor in a digital camera. Edwin Land proposed, in 1964, a complex algorithm involving image paths to compute relative lightness values from the absolute values captured by the camera [6].

Later, he published [7] an alternative formulation of the original algorithm in which the relative lightness was computed as the ratio between the value of a pixel and the weighted average of the surrounding values. This operation was followed by a logarithmic mapping of the ratios in order to mimic the non-linear behaviour of the HVS with respect to low and high values of the illumination.

\footnotetext{
${ }^{1}$ https://doi.org/10.5201/ipol.2021.391
} 
A generalization of this formulation leads to the following model for the computation of the image lightness $L$

$$
L(\boldsymbol{x})=f\left(\frac{I(\boldsymbol{x})}{F * I(\boldsymbol{x})}\right)
$$

where $I(x)$ is the light intensity captured by the camera at pixel $\boldsymbol{x}$ (we are considering here a single-channel image), $F$ is a surround radial kernel, and $f$ is a global scaling function.

Equation (1) defines a Center/Surround model of image perception. In this paper we shall analyze different alternatives both for the kernel and the scaling function, and we will give algorithmical details of the implementation of the model. For a more detailed analysis we refer the reader to $[10]$.

The paper is organized as follows: in Section 2 different alternatives for the kernel function are analyzed; similarly, several possible scaling functions are examined in Section 3. The main center/surround algorithm is described in Section 4, together with some technical details. Several experiments are displayed in Section 5, and some conclusions are drawn in Section 6.

\section{Kernel Functions}

Mathematically, two conditions should be imposed to the kernel: scale invariance and integrability. As proved in [14], the only radial kernel which is scale invariant is $F(r)=\frac{K}{r^{2}}$, which is the (nonintegrable) kernel proposed by Land in [7]. Several other radial kernels have been proposed in the literature $[7,13,5,4,3,12,14,17]$. In [10] an exhaustive analysis of these kernels is performed, and some new ones are proposed. The authors unify all these kernels into five models, which are summarized in Table 1 and described next:

- $F_{A G}$ is the generalization of the Gaussian kernel and a linearized version of the multiscale Retinex (MSR) [4]. It is a weighted average of Gaussian kernels at different scales and it is defined as

$$
F_{A G}(r)=\sum_{i=1}^{N} w_{i} F_{G, \sigma_{i}}=\sum_{i=1}^{N} w_{i} \frac{1}{2 \pi \sigma_{i}^{2}} e^{-\frac{r^{2}}{2 \sigma_{i}^{2}}}
$$

where $N$ is the number of scales, $\sigma_{i}$ is the scale parameter of the $i$-th kernel and $w_{i}$ is its corresponding weight factor.

Following [4], the scales are distributed following a geometric series

$$
\sigma_{i}=\sigma_{1} r^{i-1}
$$

The initial scale $\sigma_{1}$ is a parameter. Since convolutions with Gaussians whose $\sigma$ is of the order of the image size or bigger yield a constant result, the final scale $\sigma_{N}$ can be chosen depending on the image dimensions

$$
\sigma_{N}=S \cdot \min (\text { image width, image height })
$$

where $S$ is also a parameter.

Finally, we propose to define the weights as $w_{i}=\frac{1}{N}$. In this way, we have reduced the parameters of the kernel to $N, \sigma_{1}$ and $S$. 
- $F_{I G}$ was first introduced in [14] to find a compromise between scale invariance and integrability. It is a continuous average of Gaussians from scale $\sigma_{1}$ to $\sigma_{2}$.

$$
F_{I G}(r)= \begin{cases}\frac{1}{2 \pi \log \left(\sigma_{2} / \sigma_{1}\right)} \frac{\exp \left(-\frac{r^{2}}{2 \sigma_{2}^{2}}\right)-\exp \left(-\frac{r^{2}}{2 \sigma_{1}^{2}}\right)}{r^{2}}, & r \neq 0, \\ \frac{\sigma_{1}^{-2}-\sigma_{2}^{-2}}{4 \pi \log \left(\sigma_{2} / \sigma_{1}\right)}, & r=0 .\end{cases}
$$

where the initial scale $\sigma_{1}$ is a parameter, and the final scale $\sigma_{2}$ is chosen depending on the image size as in the previous case

$$
\sigma_{N}=S \cdot \min (\text { image width, image height })
$$

where $S$ is also a parameter.

- $F_{2, \sigma}$ is the continuous version of the Land kernel $F(r)=1 / r^{2}$

$$
F_{2, \sigma}(r)=\frac{C}{\left(\frac{r}{\sigma}\right)^{2}+1},
$$

where $C$ is a normalization constant such that $\int_{\Omega} F_{2, \sigma}(r) d r=1$.

- $F_{I E}$ is a continuous average of exponentials

$$
F_{I E}(r)= \begin{cases}\frac{1}{2 \pi\left(\sigma_{2}-\sigma_{1}\right)} \frac{e^{-\frac{r}{\sigma_{2}}}-e^{-\frac{r}{\sigma_{1}}}}{r} & r \neq 0, \\ \frac{1}{2 \pi \sigma_{2} \sigma_{1}} & r=0 .\end{cases}
$$

It is inspired by an early work by Moore et al. [13], which proposed an exponential kernel

$$
F_{E, \sigma}(r)=\frac{1}{2 \pi \sigma^{2}} e^{-\frac{r}{\sigma}}
$$

that permitted a real-time implementation in analog VLSI.

In $F_{I E}$ the weight of the central point in the convolution kernel is of order $\mathcal{O}\left(\frac{1}{\sigma_{1} \sigma_{2}}\right)$ showing the inverse proportionality of both parameters. As is analyzed in [10], to obtain good results the product between $\sigma_{1}$ and $\sigma_{2}$ must be constant. Since the kernel is not scale invariant, the values of the initial and final scales $\sigma_{1}$ and $\sigma_{2}$ are chosen depending on the image size as

$$
\begin{aligned}
& \sigma_{1}=s / \min (\text { image width, image height), } \\
& \sigma_{2}=S \cdot \min (\text { image width, image height), }
\end{aligned}
$$

where $s$ and $S$ are parameters.

- $F_{1, \sigma}$ is the continuous version of the ACE kernel [17]

$$
F_{1, \sigma}(r)=\frac{C}{\left(\frac{r}{\sigma}\right)+1}
$$


where $C$ is a normalization constant such that $\int_{\Omega} F_{1, \sigma}(r) d r=1$.

This kernel is not scale invariant, therefore the scale $\sigma$ is chosen depending on the image size as

$$
\sigma=s / \min (\text { image width, image height), }
$$

where $s$ is a parameter.

Kernel

$$
\begin{aligned}
& F_{A G}(r)=\sum_{i=1}^{N} w_{i} F_{G, \sigma_{i}} \\
& F_{I G}(r)= \begin{cases}C \frac{\exp \left(\frac{-r^{2}}{2 \sigma_{2}^{2}}\right)-\exp \left(\frac{-r^{2}}{2 \sigma_{1}^{2}}\right)}{r^{2}}, & r \neq 0 \\
C \frac{\sigma_{1}^{-2}-\sigma_{2}^{-2}}{2} & r=0\end{cases} \\
& C=\frac{1}{2 \pi \log \left(\sigma_{2} / \sigma_{1}\right)}
\end{aligned}
$$

\section{$N, \sigma_{1}, S$}$$
F_{I E}(r)=\left\{\begin{array}{lll}
C \frac{\exp \left(\frac{-r}{\sigma_{2}}\right)-\exp \left(\frac{-r}{\sigma_{1}}\right)}{r}, & r \neq 0 & \begin{array}{l}
s, S \\
\sigma_{1}=s / \text { min. image dimension } \\
\end{array} \\
\frac{1}{2 \pi \sigma_{2} \sigma_{1}}, & r=0 &
\end{array}\right.
$$$$
C=\frac{1}{2 \pi\left(\sigma_{2}-\sigma_{1}\right)}
$$

$\sigma_{1}=s /$ min. image dimension

$\sigma_{2}=S \cdot$ min. image dimension

$$
F_{1, \sigma}(r)=\frac{C}{\left(\frac{r}{\sigma}\right)+1} \quad \begin{aligned}
& s \\
& \sigma=s / \text { min. image dimension }
\end{aligned}
$$

$$
F_{2, \sigma}(r)=\frac{C}{\left(\frac{r}{\sigma}\right)^{2}+1}
$$

Table 1: The five models of surround kernels that summarize the state of the art. 


\section{Optimum Values of the Kernel Parameters}

In [10] the authors studied, in terms of some distortion measures, the range of values of the parameters of the different kernels. In this study they observed that kernels $F_{A G}, F_{I G}$ and $F_{2, \sigma}$, which are differentiable at $r=0$, produce more halo distortion than $F_{I E}$ and $F_{1, \sigma}$ which are non-differentiable at $r=0$. This is because the halo becomes more visible as the size of the kernel decreases, and the non-differentiable kernels have a less local behavior. Table 2 presents the recommended parameter values. In Section 5 several examples illustrate how these parameters influence the obtained results.

\begin{tabular}{c|c} 
Kernel & Recommended parameter values \\
\hline$F_{A G}$ & $N=5, S \geq 0.5, \sigma_{1}$ indifferent \\
\hline$F_{I G}$ & $S \geq 1, \sigma_{1} \geq 0.25$ \\
\hline$F_{I E}$ & $S \geq 0.5, s \geq 0.5$ \\
\hline$F_{2, \sigma}$ & $\sigma \geq 0.25$ \\
\hline$F_{1, \sigma}$ & $s \geq 0.1$
\end{tabular}

Table 2: Recommended ranges of values of the parameters of the kernels that guarantee a minimum degree of visual distortion in the results.

\section{Scaling Functions}

The human visual system (HVS) is able to discount the effect of the illumination in the perception of the scenes. The Retinex theory tries to mimic the HVS, with the center/surround formulation. Moreover, Land in [8] observed that the function which relates reflectance with lightness sensation can be approximated by a logarithmic function. Naka and Rushton [15] were the first to model the contrast sensitivity as a sigmoid function. Reinhard in [16] noticed the mathematical properties of sigmoid functions to model the HVS. On the other hand digital images suffer several camera processes, such as gamma-corrections and white balance, which these mapping functions must take into account. In [10] different options for these mapping functions (also known as scaling functions) were analyzed. Table 3 summarizes them. Note that these functions are defined for single channel images. For color images they are applied on a channel-by-channel basis.

In what follows, Min and Max refer to the minimum and maximum values of the range of the center/surround operation $\frac{I}{F * I}$, which shall be mapped to the usual $[0,255]$ range.

\begin{tabular}{c|c} 
Name & Function $f$ \\
Linear & $f_{L}(x)=255\left(\frac{x-\text { Min }}{\text { Max }- \text { Min }}\right)$ \\
Logarithmic & $f_{l o g}(x)=\frac{255}{\log (\text { Max }- \text { Min }+1)} \log (x-$ Min +1$)$ \\
Naka-Rushton & $f_{\alpha}(x)=255\left(\frac{x-\text { Min }}{\text { Max }- \text { Min }}\right)^{\alpha}$ \\
Histogram based & $f_{N R}(x)=\frac{255(\text { A } 1)(x-\text { Min })}{A(\text { Max }- \text { Min })+x-M i n}$ \\
\end{tabular}

Table 3: Scaling functions reviewed in Section 3. 
- Linear mapping. It consists in just a linear stretching of the input range to the desired output range.

$$
f_{L}(x)=255\left(\frac{x-\text { Min }}{\text { Max }- \text { Min }}\right) .
$$

- Logarithmic mapping. Inspired by the tone curve proposed by Drago et al. [1], which produces better results (less noisy in dark areas) than simply replacing the linear values by logarithms in Equation (8).

$$
f_{\log }(x)=\frac{255}{\log (\operatorname{Max}-\operatorname{Min}+1)} \log (x-\operatorname{Min}+1) .
$$

- Power function.

$$
f_{\alpha}(x)=255\left(\frac{x-\operatorname{Min}}{\operatorname{Max}-\operatorname{Min}}\right)^{\alpha}
$$

The parameter $\alpha$ can take positive and negative values. In our implementation, we have the option to automatically fix $\alpha$ so that the median of the input values is mapped to the center of the output range, i.e. $127.5^{2}$.

- Naka-Rushton function. Naka and Rushton [15] modeled the contrast sensitivity in the human visual system as the sigmoid function $f(x)=\frac{x}{x+s}$, where $s$ is the value which is mapped to 0.5. We modify this function such that Min is mapped to 0 and Max is mapped to 255.

$$
f_{N R}(x)=\frac{255(A+1)(x-M i n)}{A(M a x-M i n)+x-M i n},
$$

where $A>0$ is a constant that controls the steepness of the function. For large values of $A$ $f_{N R}$ tends to a linear function $f_{L}$.

In our implementation, we have the option to automatically fix $A$ so that the median of the input values is mapped to the center of the output range, i.e. $127.5^{3}$. Note that this can only be achieved for positive values of $A$ when the median is below $\frac{\operatorname{Min}+\operatorname{Max}}{2}$. If this is not the case, $A$ is fixed automatically to a large value, so $f_{N R}$ becomes linear.

- Taking into account the shape of the histogram of the center/surround operation $\frac{I}{F * I}$, we consider a histogram-based function, which is adapted to the pixel distribution of the values. It is a generalization of the one proposed by Mai et al. [11]. The function is computed as the minimizer of the functional

subject to the condition

$$
\min _{g} \int_{\text {Min }}^{\operatorname{Max}} \frac{h(x)}{g^{\prime}(x)^{p}} d x,
$$

$$
\int_{M i n}^{M a x} g^{\prime}(x) d x=255,
$$

where $h$ denotes the normalized histogram and $p>0$ is a parameter.

The closed-form solution of the minimizing problem is

$$
f_{E}(x)=C \int_{\text {Min }}^{x} h(t)^{\frac{1}{p+1}} d t
$$

where $C$ is such that $f_{E}(\operatorname{Max})=255$. Observe that for $p=0 f_{E}$ is a histogram equalization. In general, the parameter is fixed to $p=2$, as proposed in [11].

\footnotetext{
${ }^{2}$ In our implementation, if the automatically computed $\alpha$ is smaller than 0.3 , then its value is clipped to 0.3 , to prevent excessive enhancement of low input values.

${ }^{3}$ In our implementation, if the automatically computed $A$ is smaller than 0.1 , then its value is clipped to 0.1 , to prevent excessive enhancement of low input values.
} 
Definition of $\mathrm{Min}$ and $\mathrm{Max}$. The values defining the range of the center/surround operation $\frac{I}{F * I}$ could simply be computed as the minimum and maximum values of this operation. However, in order to increase the dynamic range of the final output, it is sometimes useful to clip to either 0 (black) or 255 (white) some of the lowest (resp. highest) values of the original range. Therefore, Min and Max are computed by selecting the percentages of image pixels that will be clipped to black $\left(s_{\text {black }} \%\right)$ or white $\left(s_{\text {white }} \%\right)$. Algorithm 1 describes how these values are computed. This algorithm is an adaptation of the algorithm for robust normalization proposed in [2], which itself is a variant of the simplest color balance algorithm proposed in [9].

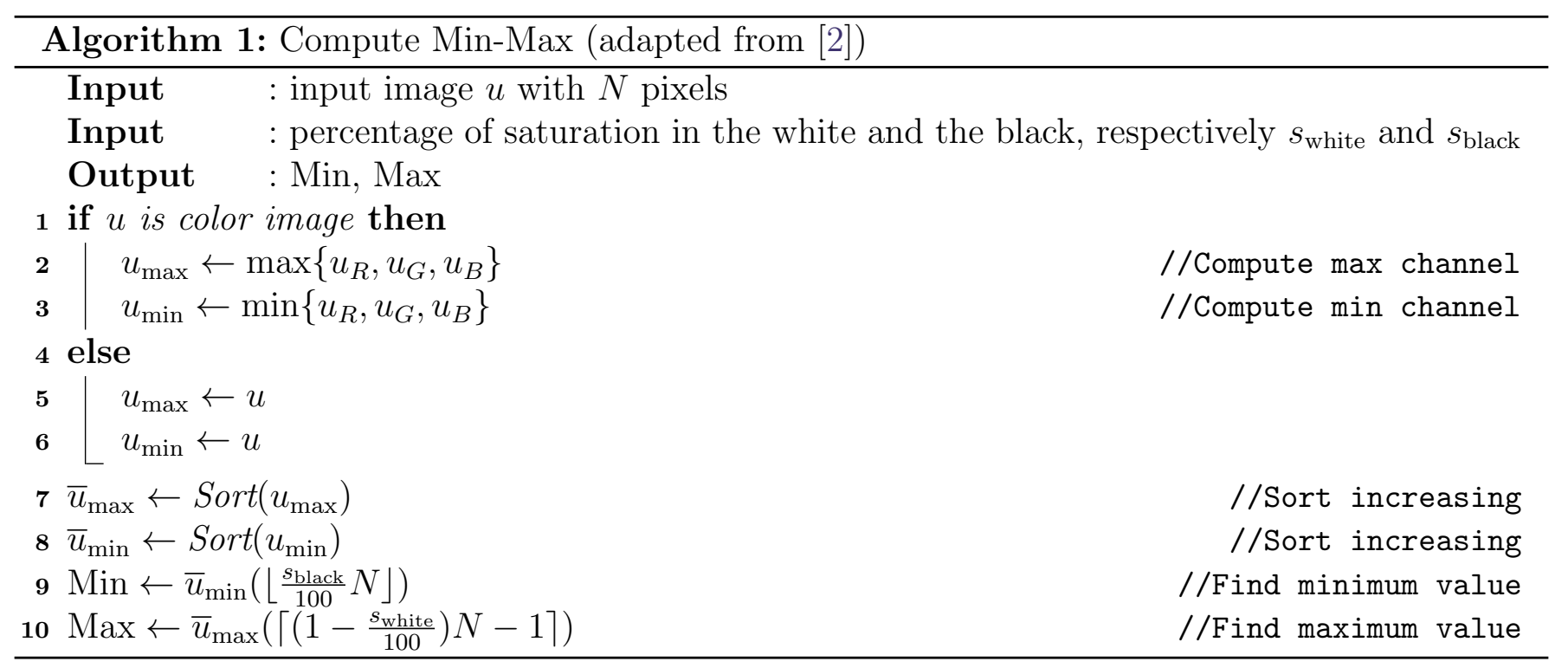

\section{Center/Surround Algorithm}

The complete algorithm for the computation of the center/surround operation on an image is presented in Algorithm 2.

Computation of the convolution $I * F$. The convolution between the original image and the kernel function is computed in the Fourier domain. By using the FFT and IFFT functions of the FFTW software library ${ }^{4}$ the original image is extended symmetrically across its sides, so that the extended image, which is four times bigger, becomes symmetric and periodic. In fact, with the FFTW library the symmetrization is implicit and performed directly as a cosine transform. By using these functions the convolution is computed as

$$
I * F=\operatorname{IFFT}\{\operatorname{DFT}(I) \cdot \operatorname{DFT}(F)\} .
$$

\section{$5 \quad$ Experiments}

In a previous article [10], we carried out an analysis for different combinations of kernel and global mappings, using quantitative and qualitative criteria, over HDR images. For these images, most of the values were concentrated on the lower part of their dynamic range. This is not usually the case for the LDR images studied in the current paper. For this reason the conclusions of our analysis

\footnotetext{
${ }^{4}$ M. Frigo and S. G. Johnson. FFTW package. http://www.fftw.org/
} 


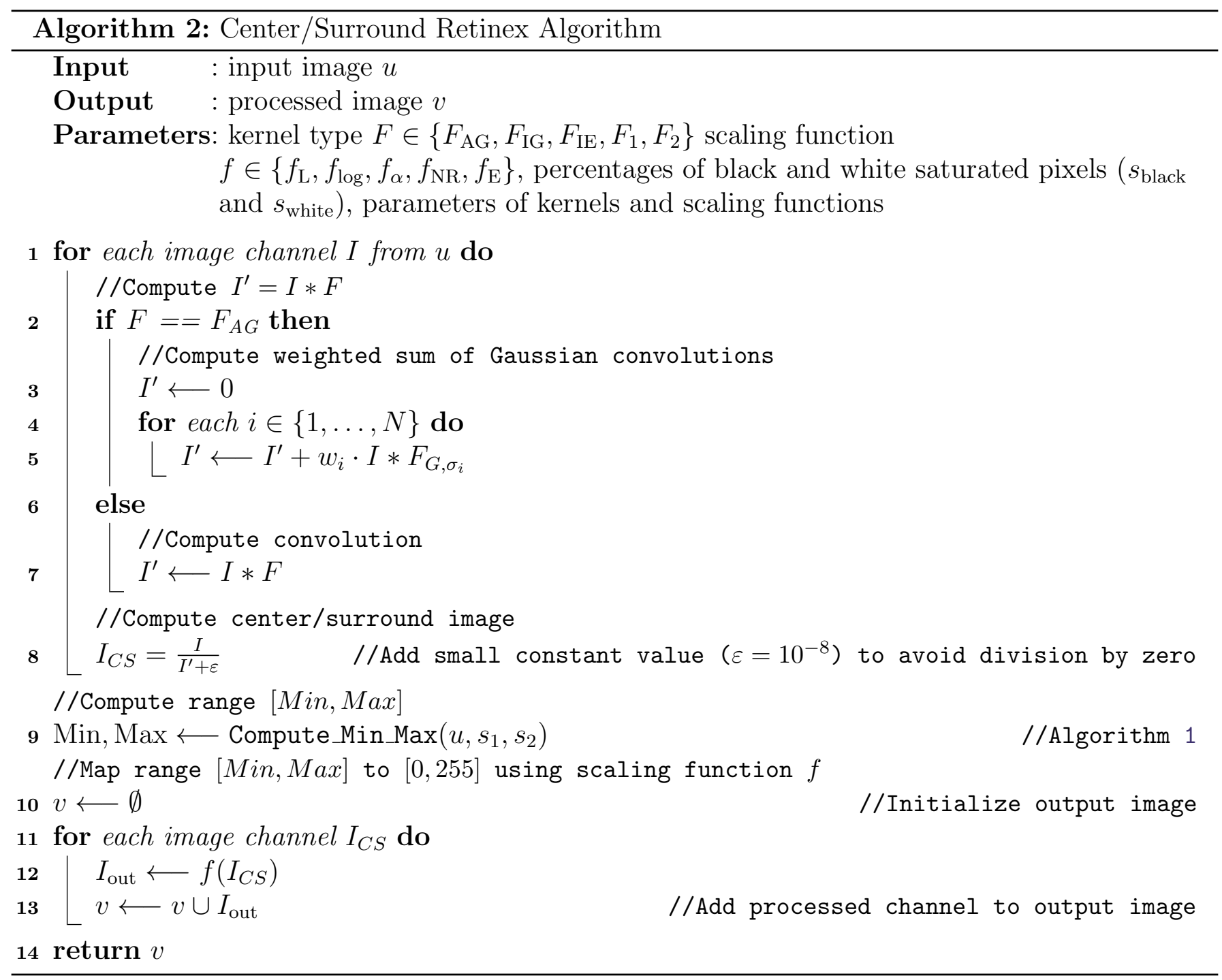

are slightly different from the ones in [10]. In general, we have observed that, for these images, the recommended ranges of values of the parameters of the kernels, shown in Table 2, are correct, but some minimum values must be increased.

In Figure 1 we compare the value of different parameters for the $F_{A G}$ kernel, with a fixed scaling function $\left(f_{\text {log }}\right)$. In this image, we can observe that we obtain better results with the number of scales greater or equal to 5 . We also observe that values of $S$ smaller than 0.5 produce artifacts in the image. The tests show that the variation of the $\sigma_{1}$ parameter does not produce meaningful changes in the results.

In the case of the $F_{I G}$ kernel, the minimum recommended value of the $\sigma_{1}$ parameter in Table 2 is 0.125 , however, for most LDR images it produces excessively bright results. A value greater or equal to 0.5 produces better results in this case. This fact can be observed in Figure 2.

The recommended values for the parameters of the kernel $F_{I E}$ in Table 2 are valid for the tested LDR images. However, the parameter of the kernel $F_{1, \sigma}$ must be increased. In Figure 3 , we can observe that values smaller than 0.5 may enhance excessively the bright parts of the image.

Finally, the $\sigma$ parameter for the kernel $F_{2, \sigma}$ should be set to values greater than 0.5 , as it can be seen in the experiments of Figure 4.

In Figure 5, we can compare the results obtained with the different kernels on the same input image and with a fixed scaling function $\left(f_{l o g}\right)$. The parameters of the kernels are fixed to their optimum values according to the previous comments. We observe that the non-differentiable kernels, 


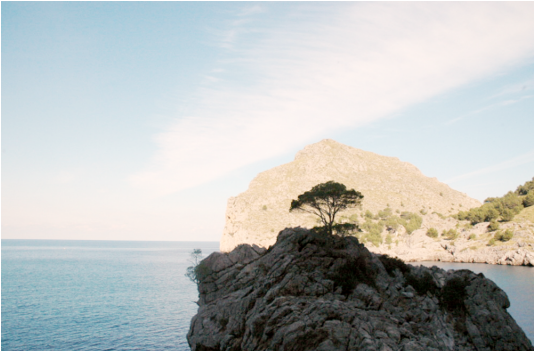

$N=2, \sigma_{1}=0.5, S=1$

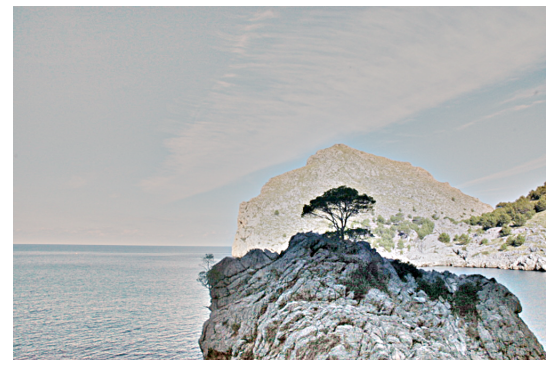

$N=5, \sigma_{1}=1, S=0.125$

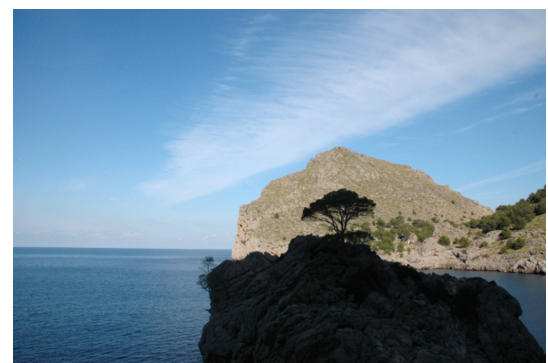

Calobra image

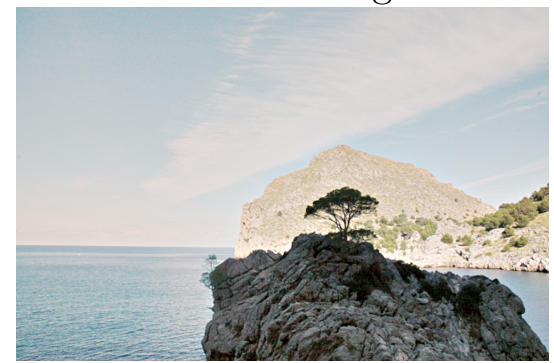

$N=5, \sigma_{1}=0.5, S=1$

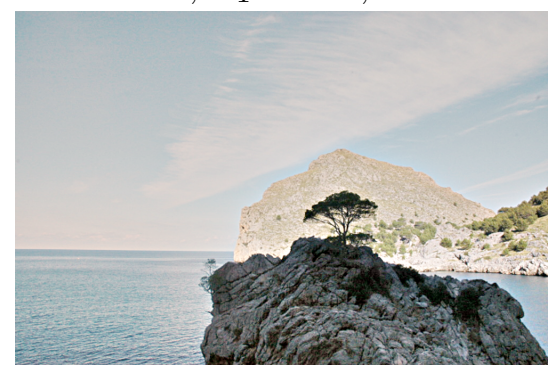

$$
N=5, \sigma_{1}=1, S=0.5
$$
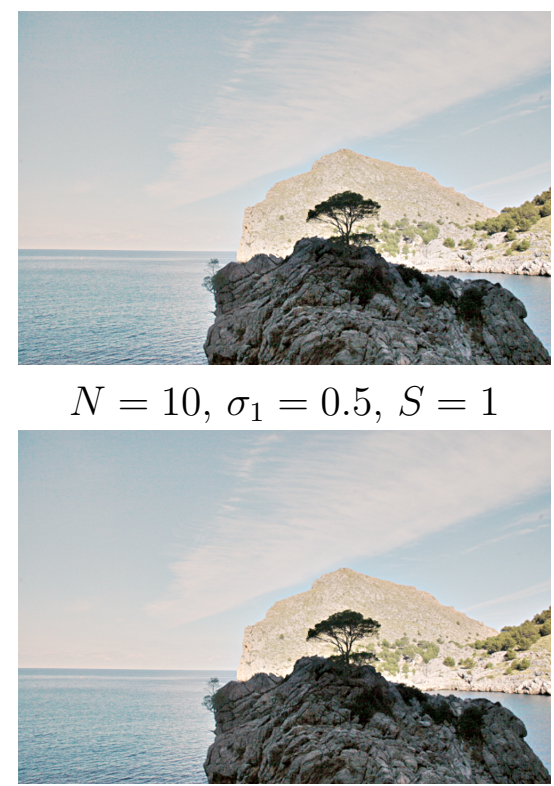

$$
N=5, \sigma_{1}=1, S=2
$$

Figure 1: Comparison between the different parameters with the $F_{A G}$ kernel with $f_{l o g}$ scaling function.

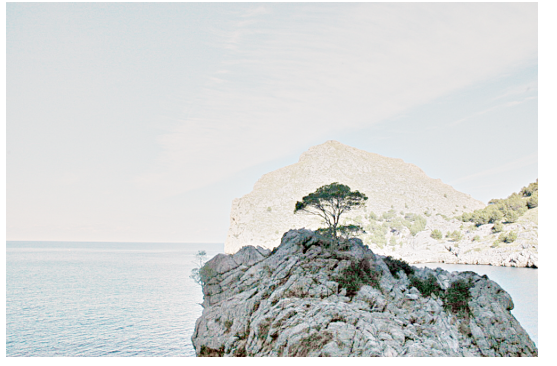

$$
\sigma_{1}=0.125, S=1
$$

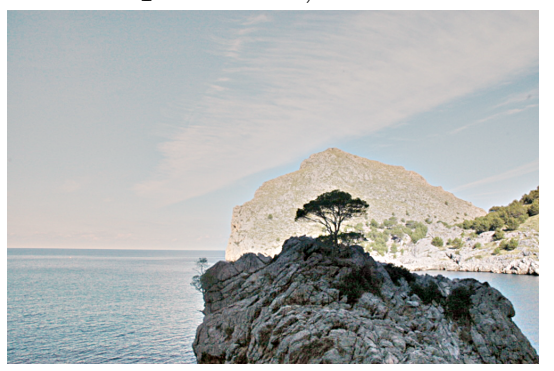

$\sigma_{1}=1, S=1$

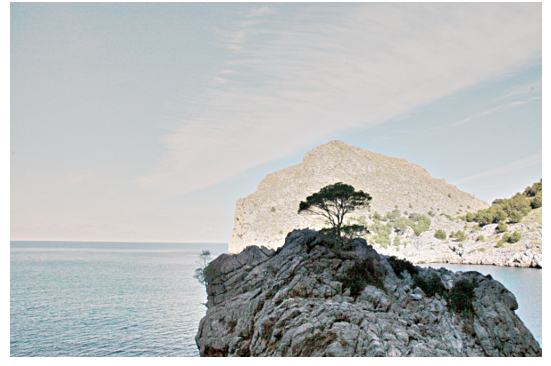

$$
\sigma_{1}=0.5, S=1
$$

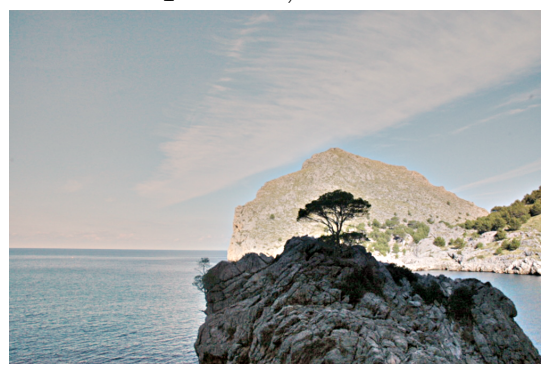

$\sigma_{1}=4, S=1$

Figure 2: Comparison between the different parameters with the $F_{I G}$ kernel with $f_{l o g}$ scaling function applied to the image Calobra in Figure 1. 


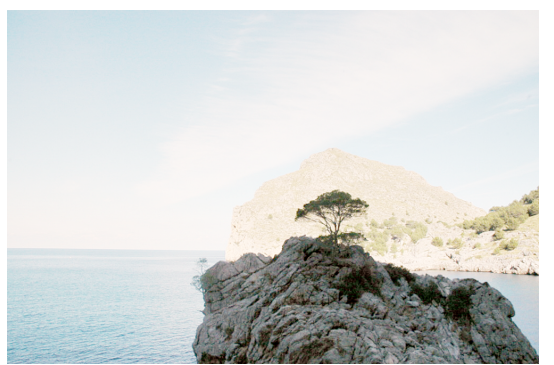

$s=0.1$

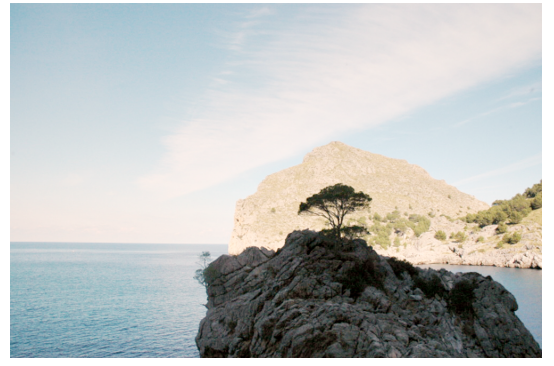

$s=0.5$

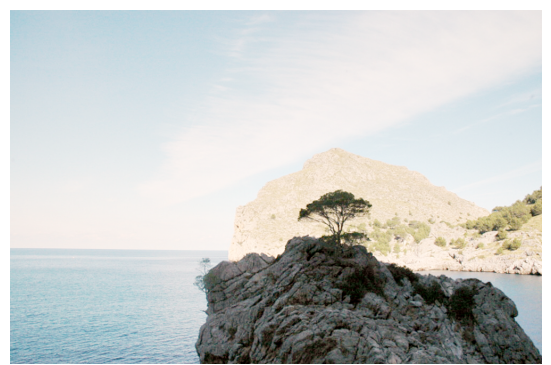

$s=0.25$

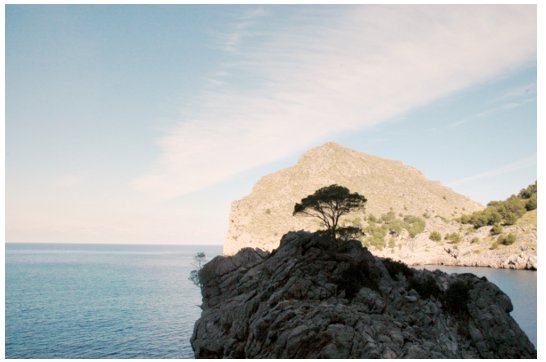

$s=1$

Figure 3: Comparison between the different parameters with the $F_{1, \sigma}$ kernel with $f_{\log }$ scaling function applied to the image Calobra in Figure 1.

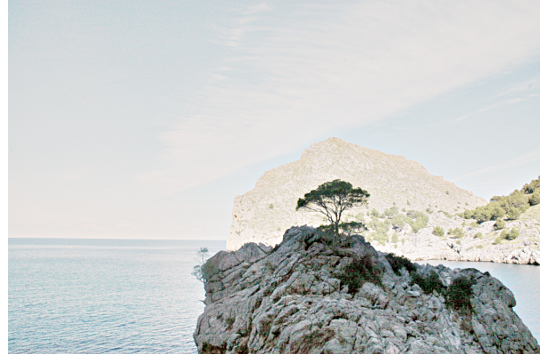

$\sigma=0.25$

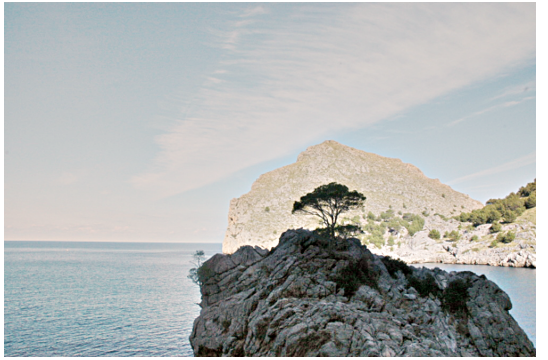

$\sigma=1$

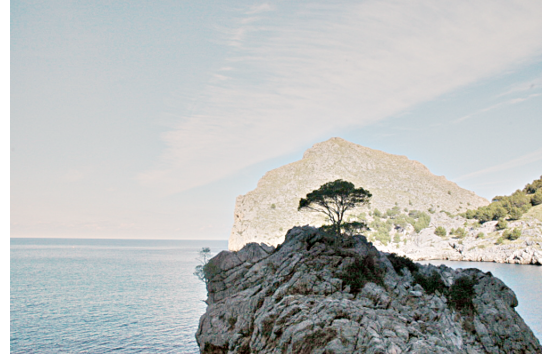

$\sigma=0.5$

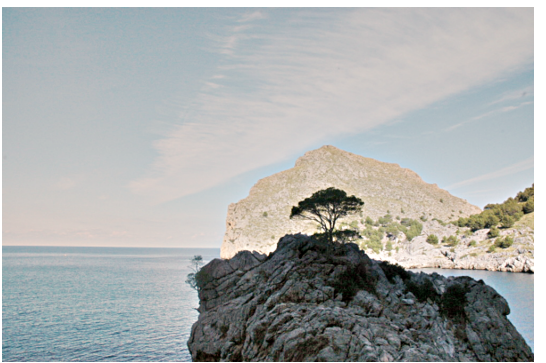

$\sigma=2$

Figure 4: Comparison between the different parameters with the $F_{2, \sigma}$ kernel with the $f_{l o g}$ scaling function applied to the image Calobra in Figure 1.

$F_{I E}$ and $F_{1, \sigma}$, obtain much better results, in terms of creation of halo artifacts. This is because the halo becomes more visible as the size of the kernel decreases, and the non-differentiable kernels have a less local behavior than the differentiable ones. Finally, in Figure 6, we can see another example. In this case, the halo artifacts are not detectable, but we can observe an excessive saturation in the window when using non-differentiable kernels.

If we analyze the scaling functions and their parameters, we observe that most of them have no parameters, or these can be fixed automatically to obtain that the median of the values of the $\mathrm{C} / \mathrm{S}$ output is mapped to 127.5 . Some results obtained with different values of the parameters for 


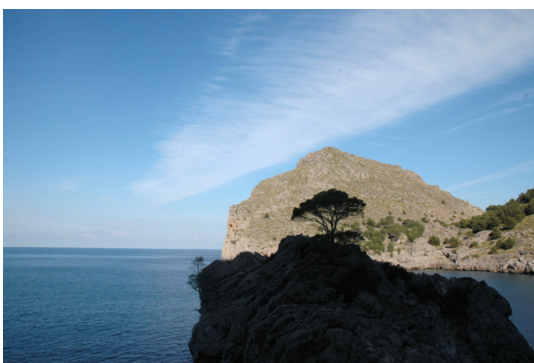

Calobra image

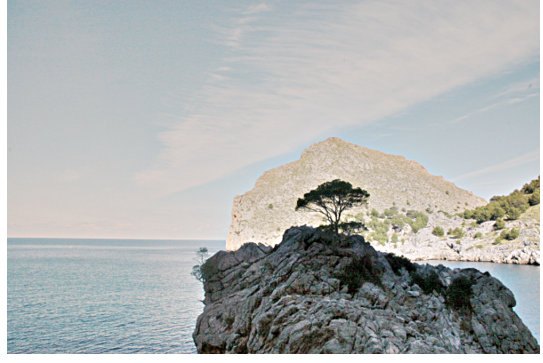

$F_{I G}$ with $S=1, \sigma_{1}=1$

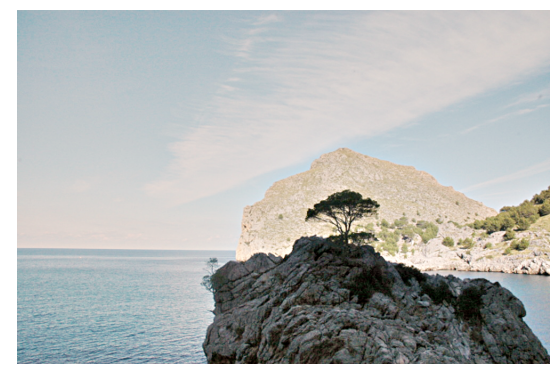

$F_{A G}$ with $N=5, \sigma_{1}=1, S=1$

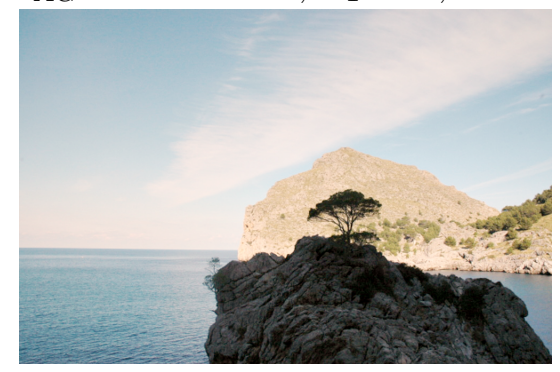

$F_{1, \sigma}$ with $s=1$

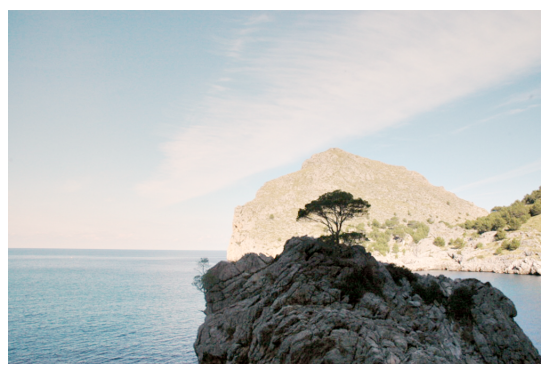

$F_{I E}$ with $S=1, s=1$

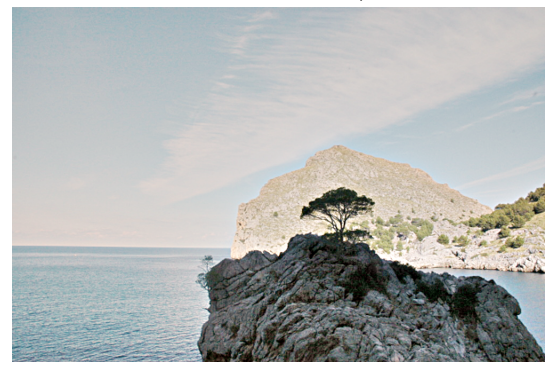

$F_{2, \sigma}$ with $\sigma=1$

Figure 5: Comparison between the different kernels with the $f_{\log }$ scaling function.

functions $f_{\alpha}$ and $f_{N R}$ are displayed in Figure 7.

Figures 8, 9 and 10 show results obtained with the different scaling functions (using the automatically estimated parameters when necessary) and three different kernels. In general, the results are brighter for $f_{l o g}$ and $f_{N R}$, and better contrasted for $f_{E}$. In some images (e.g. Figure 9 ) the results with the $f_{\alpha}$ scaling are excessively dark.

\section{Conclusions}

The center/surround formulation of the Retinex theory has been thoroughly analyzed in this paper. Several alternatives, both for kernel and scaling functions, have been considered.

Experiments conducted on several images show that non-differentiable kernels at $r=0\left(F_{I E}\right.$ $\left.F_{1, \sigma}\right)$, being less local, produce fewer visual artifacts (e.g. halos) than differentiable ones $\left(F_{A G}, F_{I G}\right.$, $\left.F_{2, \sigma}\right)$. On the other hand, the rendition of image details is better for the latter. Regarding the scaling functions, $f_{E}$ produces the better contrasted results.

The reader is invited to test the different options with the online demo associated to the article. 


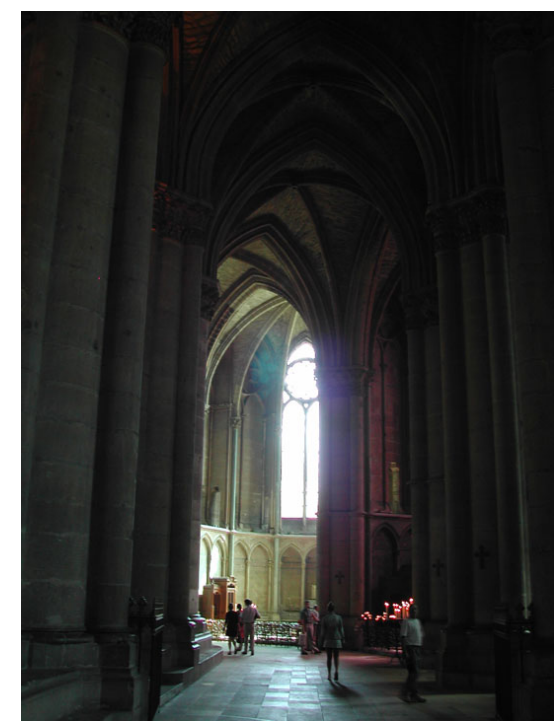

Cathedral image

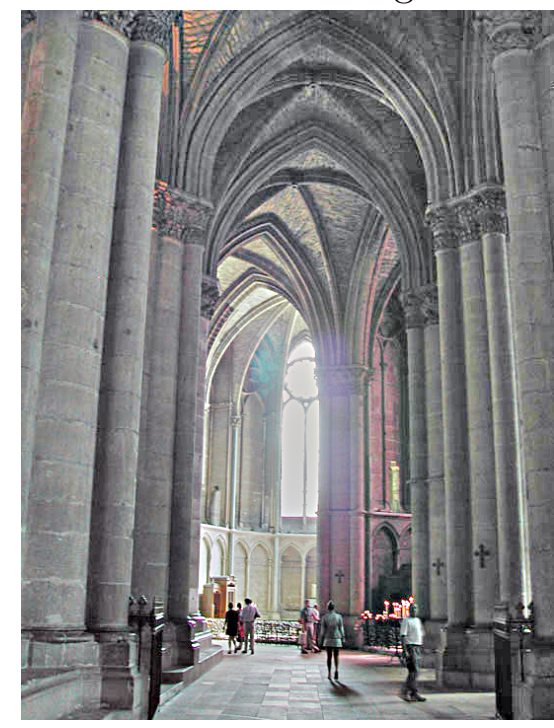

$F_{I G}$ with $S=1, \sigma_{1}=1$

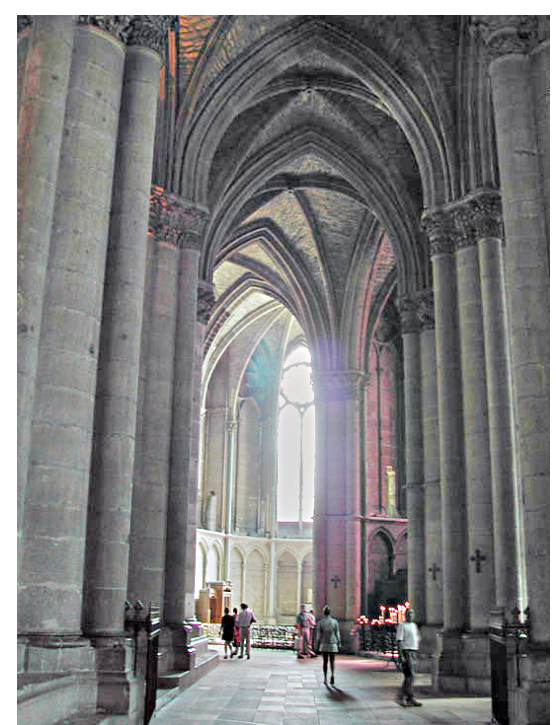

$F_{A G}$ with $N=5, \sigma_{1}=1, S=1$

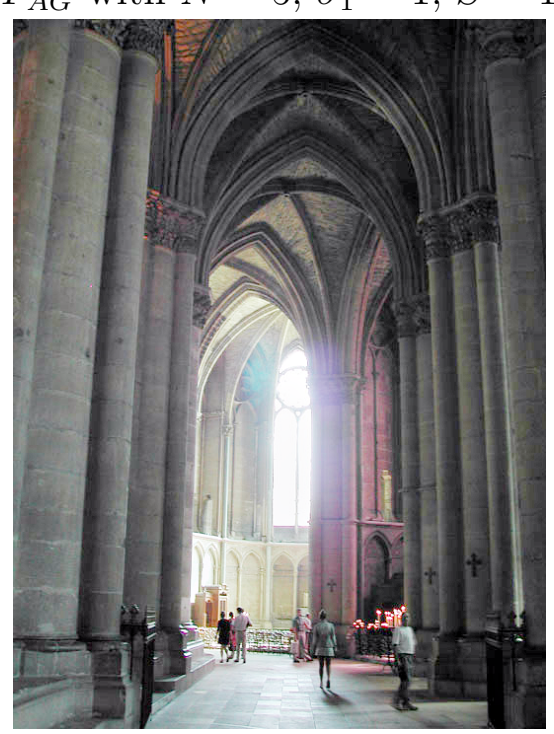

$F_{1, \sigma}$ with $s=1$

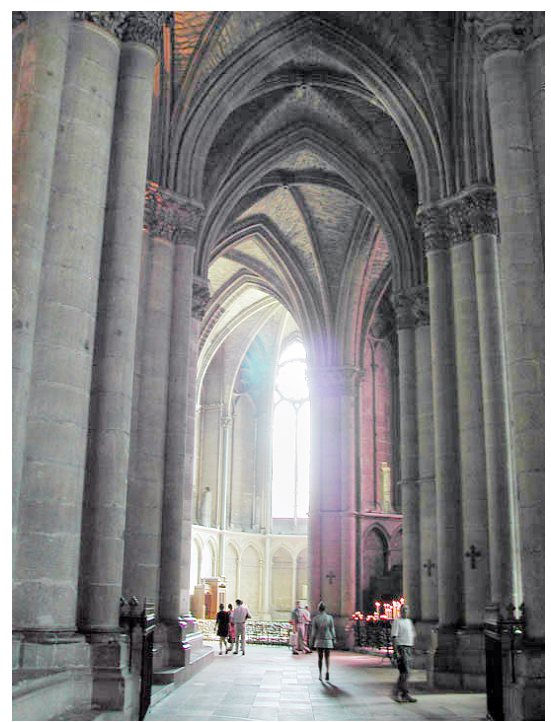

$F_{I E}$ with $S=1, s=1$

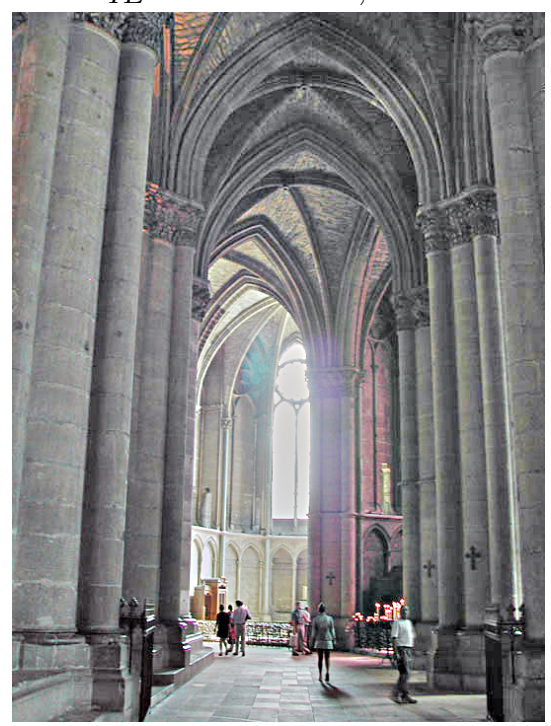

$F_{2, \sigma}$ with $\sigma=1$

Figure 6: Comparison between the different kernels with $f_{\text {log }}$ scaling function. 


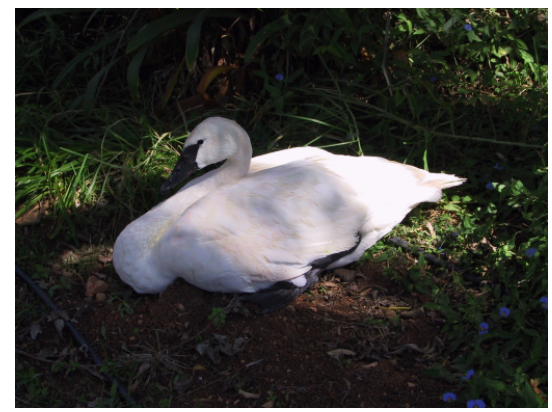

Swam image
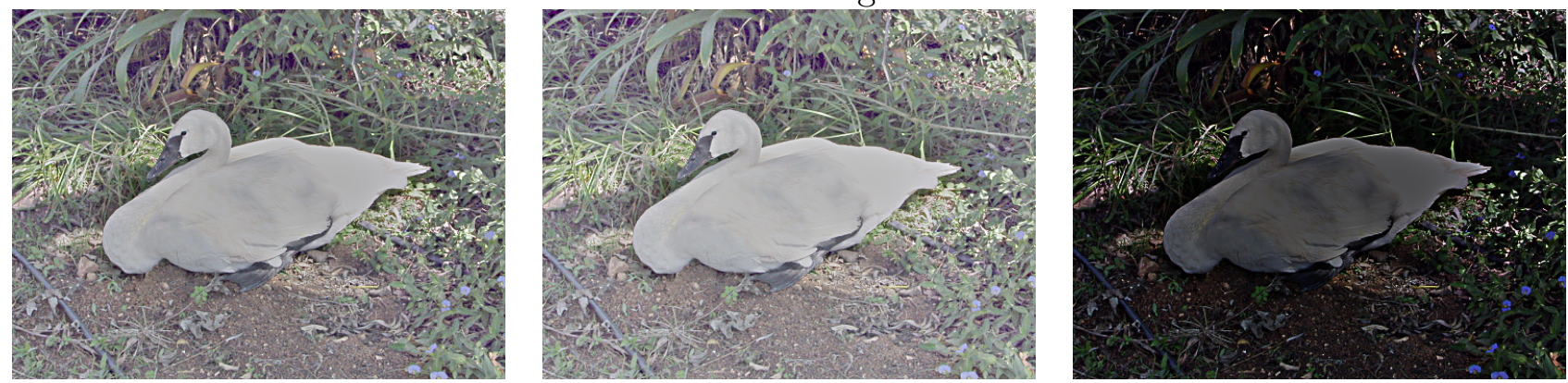

$f_{\alpha}$ with automatic value
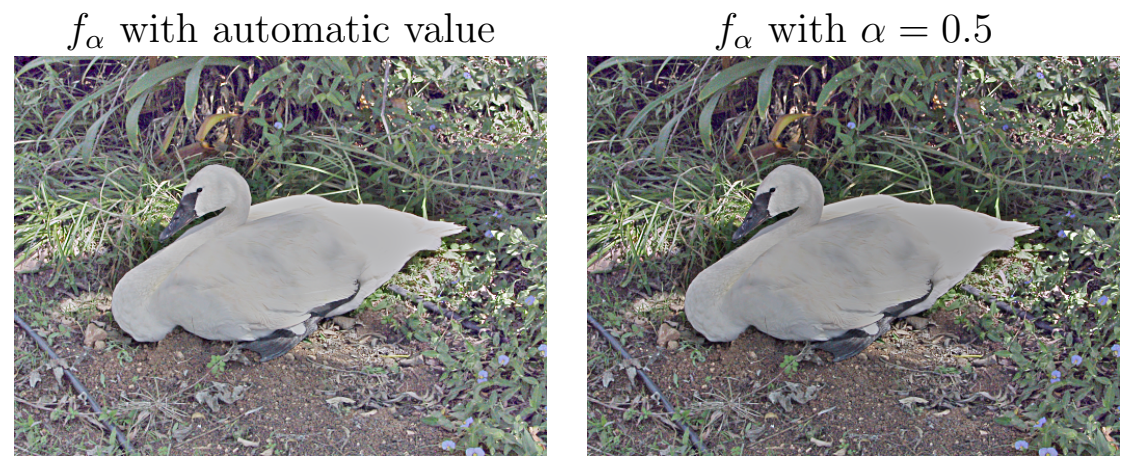

$f_{N R}$ with automatic value

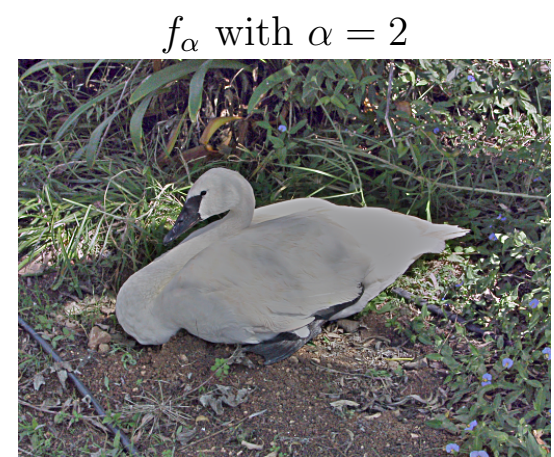

$f_{N R}$ with $A=4$

Figure 7: Comparison between the results obtained with different parameters for the $f_{\alpha}$ and $f_{N R}$ scaling functions, with fixed $F_{I G}$ kernel. 


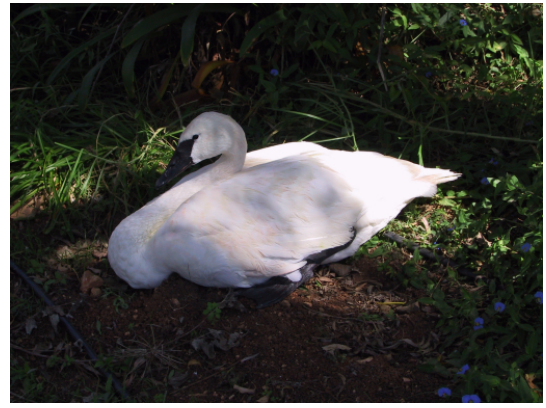

Swam image

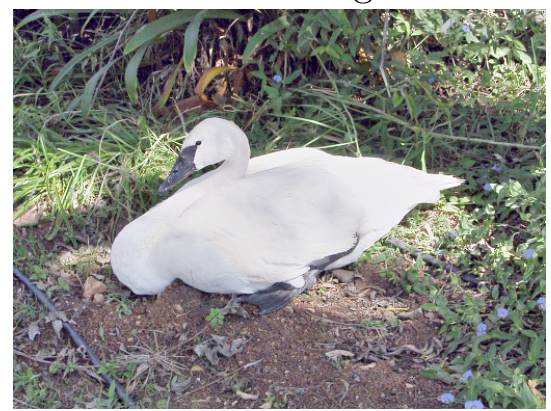

$f_{l o g}$

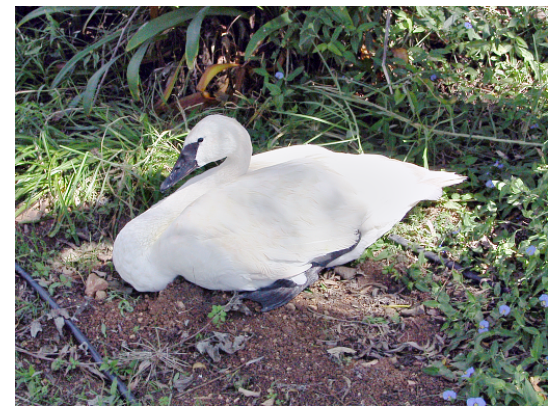

$f_{E}$ with $p=2$

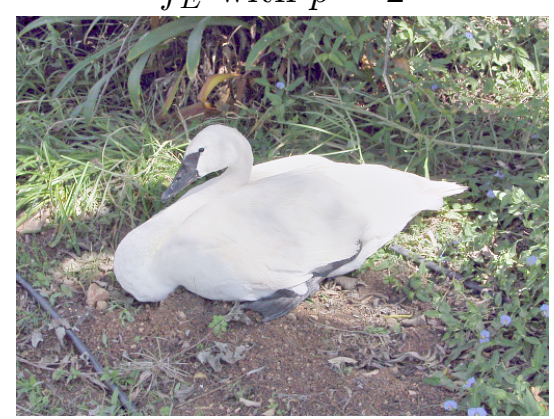

$f_{\alpha}$ with automatic value

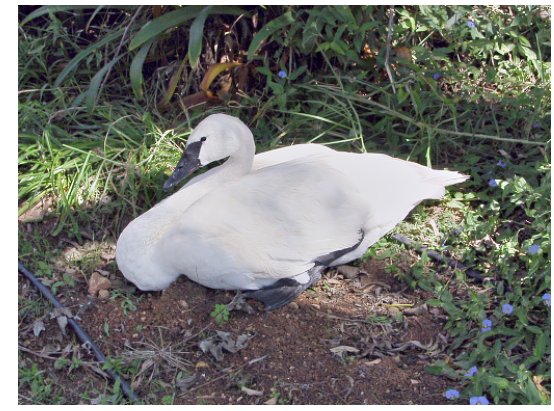

$f_{L}$

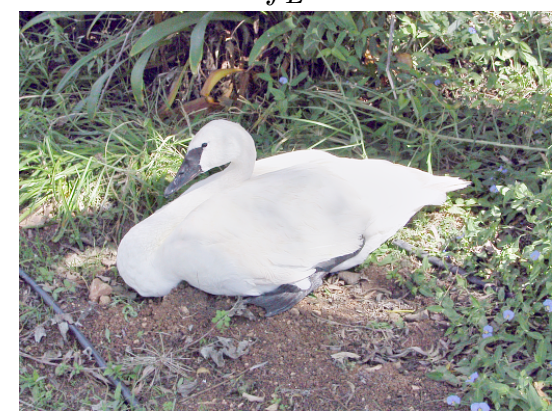

$f_{N R}$ with automatic value

Figure 8: Comparison between the results obtained with different scaling functions, using a fixed $F_{I E}$ kernel.

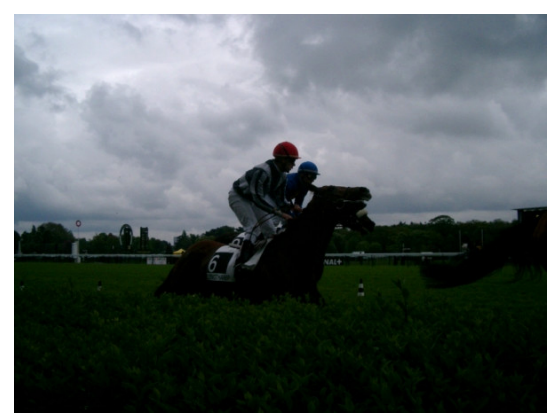

Horses image

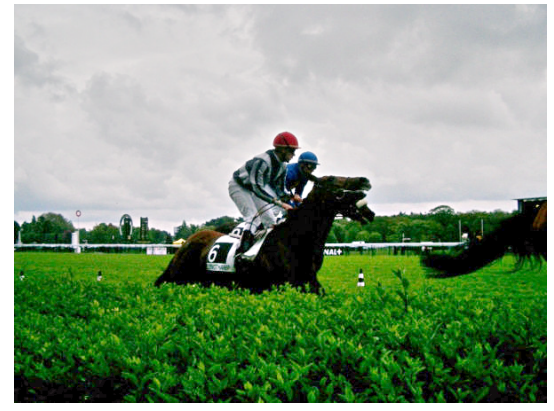

$f_{\log }$

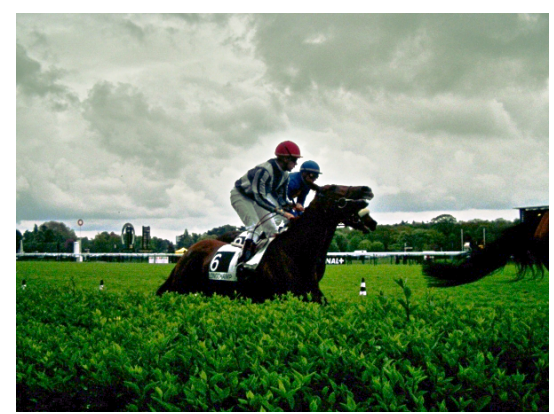

$f_{E}$ with $p=2$

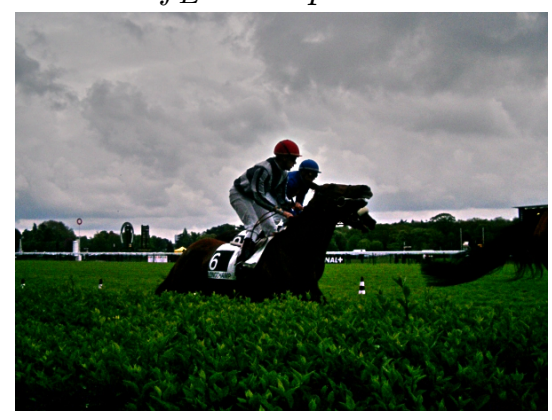

$f_{\alpha}$ with automatic value

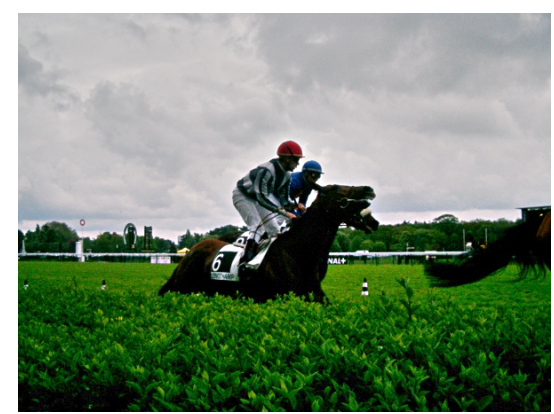

$f_{L}$

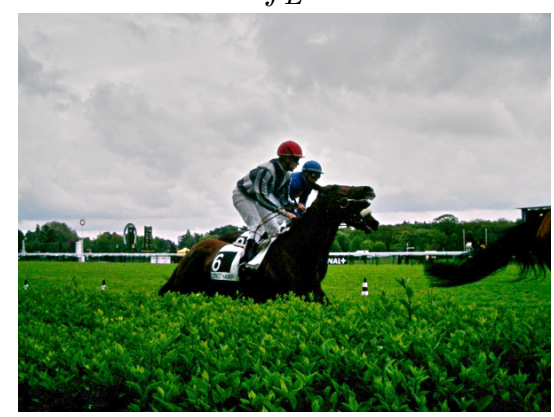

$f_{N R}$ with automatic value

Figure 9: Comparison between the results obtained with different scaling functions, using a fixed $F_{A G}$ kernel. 


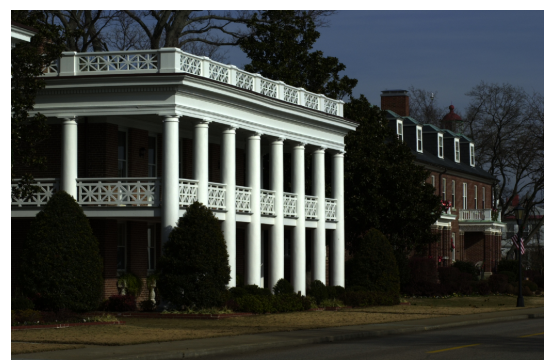

Horses image

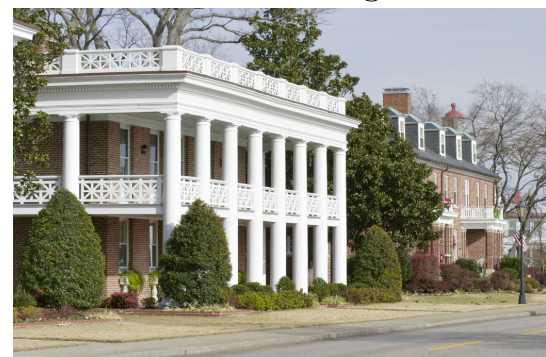

$f_{l o g}$

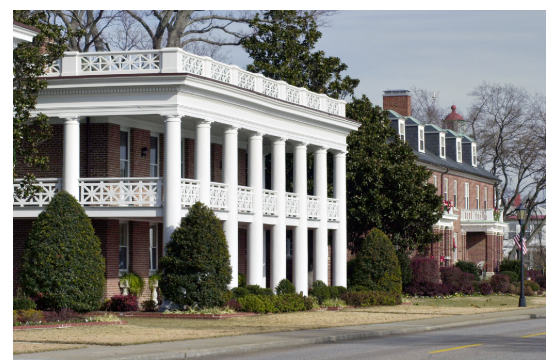

$f_{E}$ with $p=2$

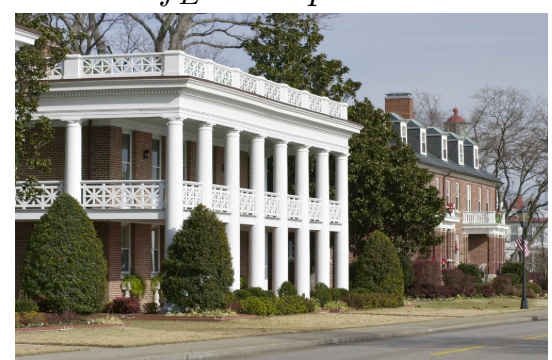

$f_{\alpha}$ with automatic value

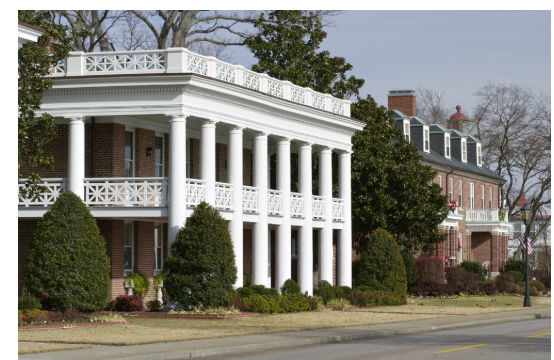

$f_{L}$

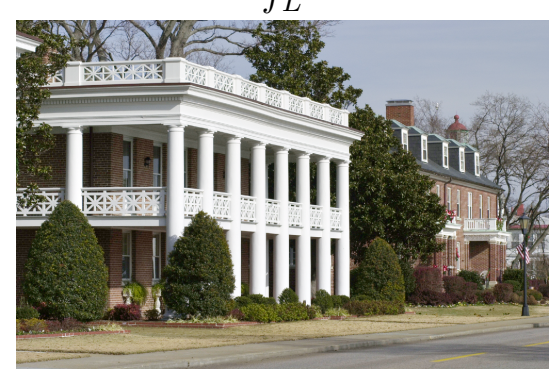

$f_{N R}$ with automatic value

Figure 10: Comparison between the results obtained with different scaling functions, using a fixed $F_{1, \sigma}$ kernel. 


\section{Acknowledgements}

The authors have been partially sponsored by MINECO/AEI/FEDER, UE projects TIN2017-85572P, DPI2017-86372-C3-3-R, and by the Comunitat Autonoma de les Illes Balears through the Direcció General de Política Universitària i Recerca with funds from the Tourist Stay Tax Law ITS 2017-006 (PRD2018/26).

\section{Image Credits}

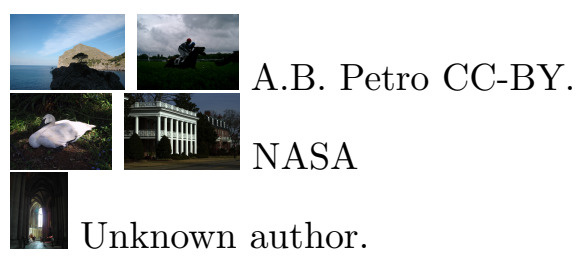

\section{References}

[1] F. Drago, K. Myszkowski, T. Annen, And N. Chiba, Adaptive logarithmic mapping for displaying high contrast scenes, Computer Graphics Forum, 22 (2003), pp. 419-426. http: //dx.doi.org/10.1111/1467-8659.00689.

[2] C. Hessel, An Implementation of the Exposure Fusion Algorithm, Image Processing On Line, 8 (2018), pp. 369-387. https://doi.org/10.5201/ipol.2018.230.

[3] A.C. Hulbert, Formal connections between lightness algorithms, Journal of the Optical Society of America, 3 (1986), pp. 1684-1693. https://doi.org/10.1364/JOSAA.3.001684.

[4] D.J. Jobson, Z. Rahman, And G.A Woodell, A multiscale retinex for bridging the gap between color images and the human observation of scenes, IEEE Transactions on Image Processing, 6 (1997), pp. 965-976. https://doi.org/10.1109/83.597272.

[5] D.J. Jobson, Z. Rahman, And G.A. Woodell, Properties and performance of a center/surround retinex, IEEE Transactions on Image Processing, 6 (1997), pp. 451-462. https: //doi.org/10.1109/83.557356.

[6] E.H. LAND, The retinex, American Scientist, 52 (1964), pp. 247-264.

[7] E.H. LAND, An alternative technique for the computation of the designator in the retinex theory of color vision, in Proceedings of the National Academy of Science, vol. 83 of 10, 1986, pp. 3078 3080. https://dx.doi.org/10.1073/pnas.83.10.3078.

[8] E. H. Land, The retinex theory of color vision, Scientific American, 237 (1977), pp. 108-128.

[9] N. Limare, J.L. Lisani, J-M. Morel, A.B. Petro, and C. Sbert, Simplest Color Balance, Image Processing On Line, 1 (2011), pp. 297-315. https://doi.org/10.5201/ipol. 2011. Ilmps-scb.

[10] J.L. Lisani, J-M. Morel, A.B. Petro, And C. Sbert, Analyzing center/surround retinex, Information Sciences, 512 (2020), pp. 741-759. https://doi.org/10.1016/j.ins.2019.10. 009. 
[11] Z. Mai, H. Mansour, R. Mantiuk, P. Nasiopoulos, R. K. Ward, and W. HeiDRICH, Optimizing a tone curve for backward-compatible high dynamic range image and video compression, IEEE Transactions on Image Processing, 20 (2011), pp. 1558-1571. http: //dx.doi.org/10.1109/TIP.2010.2095866.

[12] L. Meylan and S. Süsstrunk, Bio-inspired color image enhancement, in SPIE, vol. 5292, 2004, pp. 46-56. http://dx.doi.org/10.1117/12.526545.

[13] A. Moore, J. Allman, and R. M. Goodman, A real-time neural system for color constancy, IEEE Transactions on Neural Networks, 2 (1991), pp. 237-246. https://doi.org/10.1109/ 72.80334.

[14] J. M. Morel, A. B. Petro, And C. Sbert, What is the right center/surround for Retinex?, in IEEE International Conference on Image Processing (ICIP), 2014, pp. 4552-4556. https: //doi.org/10.1109/ICIP.2014.7025923.

[15] K. I. NAKA And W. A. H. Rushton, S-potentials from colour units in the retina of fish (cyprinidae), Journal of Physiology, 185 (1966), pp. 536-555. https://dx.doi.org/10.1113/ jphysiol.1966.sp008001.

[16] E. Reinhard, G. Ward, S. Pattanaik, and P. Debevec, High Dynamic Range Imaging: Acquisition, Display, and Image-Based Lighting (The Morgan Kaufmann Series in Computer Graphics), Morgan Kaufmann Publishers Inc., San Francisco, CA, USA, 2005. ISBN 0125852630.

[17] A. Rizzi, C. Gatta, And D. Marini, A new algorithm for unsupervised global and local color correction, Pattern Recognition Letters, 24 (2003), pp. 1663-1677. https://doi.org/10.1016/ S0167-8655(02)00323-9. 